\title{
Is Communicating Material an Intelligent Product instantiation? Application to the McBIM project
}

\author{
WAN, H., DAVID, M., DERIGENT, W.
}

*Research Centre for Automatic Control, CRAN CNRS UMR 7039, Université de Lorraine, Campus Sciences, BP 70239, 54506 Vandoeuvre-lès-Nancy, France

(e-mail: firstname.lastnameduniv-lorraine.fr)

\begin{abstract}
Information and communication technologies, like Wireless Sensor Network (WSN), allow to imagine new objects. Objects whose material could be composed of micro-elements able of data sensing, storing, processing, and communicating. Communicating Materials (CM) is a new paradigm inspired by Intelligent Product concept. This paper presents a recursive Multi-Agent Framework proposed for handling composition of Communicating Materials, which is an application of the Janus Effect. The proposed framework, still in development, is illustrated on the McBIM project, consisting in designing a communicating concrete and digital services that can be used by Building Information Modelling (BIM) applications throughout its lifecycle. MAS principles are demonstrated on this construction industry project to control WSN energy and to illustrate CM aggregation ability.
\end{abstract}

Keywords: Communicating materials, Recursive Multi-Agent System, Wireless Sensor Network, Digital Twin.

\section{Introduction and Context}

In the framework of the IMS (Intelligent Manufacturing Systems) community, the use of the Internet of Things gave rise to the concept of intelligent products. Indeed, substantial information distribution improves data accessibility and availability compared to centralized architectures. Product information may be allocated both within fixed databases and/or within the product itself, thus leading to products with informational and/or decisional abilities, referred as "Intelligent Products". Many different definitions of "Intelligent Products" have been proposed. A comparison of these different types is provided in (McFarlane, Giannikas, Wong, \& Harrison, 2013). 
In 2010, a new paradigm was proposed in (Sylvain Kubler, Derigent, Thomas, \& Rondeau, 2010), introducing "communicating materials", i.e materials able to communicate with their environment, process and exchange information, and store data in their own structure. Besides, they also have the capability to sense their environment and measure their own internal physical states.

The concept has been applied in different works, from different perspectives. Diverse early prototypes were designed (or simulated) for the needs of the manufacturing and the construction industry, by spreading micro-electronics devices into a material. The material could be either wood, textile or concrete. The interests of such material are diverse: (a) because of their data storing capacity, they can convey all information related to design, manufacturing and logistics, useful during the BOL (Beginning Of Life - design, manufacturing and construction) and the EOL (End Of Life dismantlement and recycling) of a building; (b) given their ability to sense their environment and process related information, they can also be used during the MOL (Middle Of Life - exploitation and maintenance) as intelligent building sensors, mainly to perform structural health monitoring. In our works, the inserted devices were either RFID tags (S. Kubler, Derigent, Thomas, \& Rondeau, 2014) or self-powered wireless sensor networks (WSNs) embedded into the material (Mekki et al., 2016). Both works deal with the data dissemination / data replication in this type of materials, which is an issue related to the second capability. However, these works did not address the issue related to the first capability, i.e. the composition/decomposition of communicating materials.

The rest of the paper is organized as follows: section 2 details the problem of composition/decomposition in communicating materials. Indeed, from a holonic perspective, it shows that composition/decomposition of communicating materials is similar to the composition/decomposition of group of product holons. Section 3 introduces the different works existing in the holonic and multi-agent literature around recursive holarchies and multi-agent systems, sometimes applied to WSN. Section 4 details the approach used to model the communicating material. Section 5 demonstrates the approach by instantiating it on the ANR McBIM (Materials Communicating with the $B I M)$ project.

\section{The Communicating Material as an infinite Holarchy of Product Holons}

\subsection{Definition of a communicating material}

Even if studied for a long time, no clear formal definitions of the communicating material have been provided until now and a first definition is proposed hereafter. This definition helps to understand that a communicating material can be theoretically considered as an infinite group of Product Holons.

In our definition, the Communicating Material is an Intelligent Product with two additional capabilities: 
- The capability of being intrinsically and wholly communicating: even if the product undergoes a physical transformation, the resulting pieces shall still be communicating materials. Two operators of physical transformation are considered: composition and decomposition. Composition is an operator that gathers 2 to $N$ communicating materials into one. Decomposition is the inverse operator, that divides one communicating materials into 2 to $N$ different pieces, still communicating materials.

- The capability of managing its own data: The material should be able to manage its own data according to the events occurring in its environment. For instance, the material could decide itself to propagate/replicate specific data onto different material parts because a physical transformation is scheduled, thus avoiding data loss.

As said before, a communicating material is a special type of intelligent product. Therefore, based on the definition of (Ventä, 2007; Wong, Mcfarlane, Zaharudin, \& Agarwal, 2002), the communicating material is a product that has part or all the following 7 characteristics:

1. Possesses a unique identity

2. Is capable of communicating effectively with its environment

3. Can retain or store data about itself

4. Deploys a language to display its features, production requirements, etc.

5. Is capable of participating in or making decisions relevant to its own destiny

6. Continuously monitors its status and environment

7. Can remain a communicating material under composition or decomposition.

Characteristics (1) to (5) are inherited from (Wong et al., 2002), Characteristic (6) is from (Ventä, 2007). This one is important since it underlines the capacity of a product to monitor its own status or properties. Characteristic (7) is the only one completely dedicated to the communicating material concept and is mandatory to build a communicating material. Let $P_{1}, P_{2}$ and $P_{3}$ be 3 products (intelligent or not). the composition operator $C$ is then defined as follows (equation 1):

$$
\left(P_{1}, P_{2}\right) \stackrel{c}{\rightarrow} P_{3}
$$

The decomposition operator $D$ is defined as the inverse of $C$.

\subsection{Communicating material and recursive holarchies}

Characteristic (7) is then invariant under operators $C$ or $D$. This underlines a central aspect of the communicating material, i.e their type is invariant under composition or decomposition operators. This is a fundamental property of Holons in general as introduced by (A Koestler, 1967). 


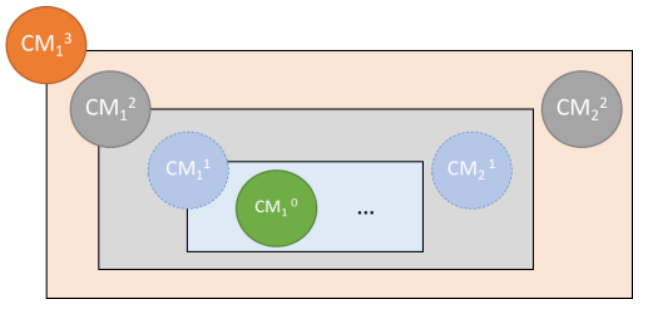

(a) Set representation

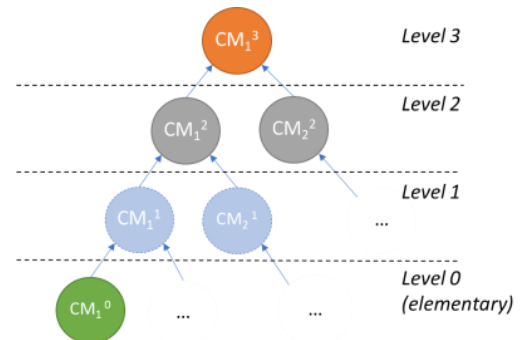

(b) Graph representation

Fig. 1. Representation of a communicating material

Indeed, our hypothesis is then to consider a communicating material as a Product Holon and as a recursive dynamical holarchy of Product Holons at the same time (which is a straightforward interpretation of the Janus Effect applied on the Product Holon (Arthur Koestler, 1979). This representation of the communicating material is one solution satisfying characteristic (7). We thus define a communicating material as a holarchy of sub-communicating material $C M_{j}{ }^{i}$, where $i$ is the level of holarchy the communicating material is attached to, and $j$ the index of the communicating material in this corresponding level Each sub-communicating material could then be decomposed in other communicating materials until a certain level of decomposition called elementary. Indeed, even if the level of the hierarchy could be infinite, it is limited in practice. The elementary level is the finest granularity of the holarchy. Different representations of a communicating material are illustrated fig. 1.

\subsection{Problem statement}

This section has highlighted a correspondence between a communicating material and a holarchy of product holons. The main problem is now to precisely describe the inner organization of this holarchy, as well as the mechanisms needed to dynamically construct this holarchy when composition and decomposition transformations occur. To do so, the next section will introduce some works dedicated to recursive multi-agent systems and recursive holarchies, sometimes applied to Wireless Sensor Nodes (as WSN are one interesting way to construct communicating materials). The concepts and notions detailed in these works will help to construct the proposal, answering to the problem statement.

\section{Review of recursive Holarchies or MAS}

In the Holonic and in MAS communities, several attempts were made to address recursion in different ways. The ANEMONA methodology, developed in (Botti \& Giret, 2008), introduces the notion of abstract agent which could be either a classic agent or a MAS (Multi-Agent Systems), itself containing agent or abstract agents. In ANEMONA, recursion is used during the design of an IMS, to define iteration after iteration the Holarchy and its corresponding levels and sub-levels. It helps to structure a given Holarchy of the future HMS. However, it does not aim to produce dynamic 
recursive Holarchies, i.e Holarchies that can evolve dynamically via composition/decomposition mechanisms through time.

In the maintenance domain, (Le Mortellec, Clarhaut, Sallez, Berger, \& Trentesaux, 2013) introduce a recursive Holarchy dedicated to product/system monitoring. In this architecture, a generic model of product Holon is defined and reused recursively in all the levels of the architecture. Each Holon owns a diagnostic function that processes the data coming from the levels above (devices or other Holons) and sending a diagnostic report to the upper level. This works stresses the need to have diagnostic functions between levels, that can be seen as filters, aggregation functions or semantic transformations between Holons of two different levels. Here also, in this paper, dynamicity in the Holarchy is neither really required nor described.

(Sallez, Montreuil, \& Ballot, 2016) defines a recursive Holarchy applied to Active Physical-Internet (PI)-containers, which are intelligent products. Each PI-container can exploit different sources of information to support its activeness (embedded static or dynamic data, measurements via sensors, etc.). Composition and encapsulation mechanisms are defined. Encapsulation refers to the action of encapsulating one container into another of a different type, whereas the composition mechanism builds a "composite" container from two containers of the same type. However, the composition mechanism is not formally defined.

The comparison of WSN with MAS is natural, as the two approaches consist of interactive entities situated in an environment they can sense and act upon locally. Agents provide engineers with a higher abstraction level, so that WSN become a useful application domain for MAS. With COSA (Coalition Oriented Sensing Algorithm), (Delgado, 2014) proposed to use a dynamic MAS to structure the possible coalitions with two kinds of agents: Leader and Follower agents. The coalition process goes through all existing stages and finishes with an agreement between the two agents. The agent initiating the dialogue assumes the leader role while its neighbor becomes its follower. COSA endows a network with self-organization capacity. This ability can be used to adapt the energy consumption of a WSN to changes in the environment and, at the same time, to fulfil sampling objectives in terms of the quality of the information reported to the sink. Very few works propose WSN MAS with dynamic capabilities (Delgado, 2014) and even fewer proposals allow to manage the different abstraction levels necessary for the applications using communicating materials.

In (Hoang, 2012), a generic recursive multi-agent system (MAS-R) is formalized. Main MAS-R principles and agents are described fig. 2. Agents $\mathrm{a}_{0}{ }^{\mathrm{i}}$ are "Elementary" agents which have a physical applicative part and a recursive agent part (holon). Agents $\mathrm{a}_{1}{ }^{\mathrm{i}}, \mathrm{a}_{2}{ }^{\mathrm{i}}$ and so on ... represent agent of higher level $i$ and are individually called "Partial" agent (no physical part). A "Complete Elementary" agent is composed by its elementary agent and all theirs directly related "Partial" agents (from abstraction levels). "Composed" agent are the gathering of few "Partial" agents from the same abstraction level. Elementary agents are dynamically added to the Holarchy. 
At the same time, depending on the states of the agents, composed agents are also automatically created or destroyed respectively via composition and reduction mechanisms. Composition occurs when one agent reaches a certain state (detected via $o b$ servation functions). In that state, it launches a negotiation protocol with the agents belonging to the same level in order to aggregate the agents into one composed agent. To generate the new composed agent and its relations with the other agents, the authors introduce the notion of transformation functions. Indeed, the VOWELS paradigm (Demazeau, 1995) states that a SMA can theoretically decomposed as \{Agents, Environment, Interaction, Organisation $\}$. As a result, 4 types of transformation functions are introduced, one for each component of the SMA, respectively $P A, P E, P I$ and $P O . P A$ is an operator grouping agents from one level and associating them to an agent of the upper level. $P E$ is an operator grouping elements of the environment from one level into an element of the environment from the upper level. $P I$ is an operator transforming interactions from agents of a level to interactions to the upper level. perceptions/actions and messages of agents of a level $N$ are transformed thanks to $P I$ to perceptions/actions and messages of agents of level $N+1$. PO is an operator that transforms relations between agents. A relation between agents of level $N$ grouped in different agents of level $N+1$ is transformed by $P O$ into a relation between agents of level $N+1$.

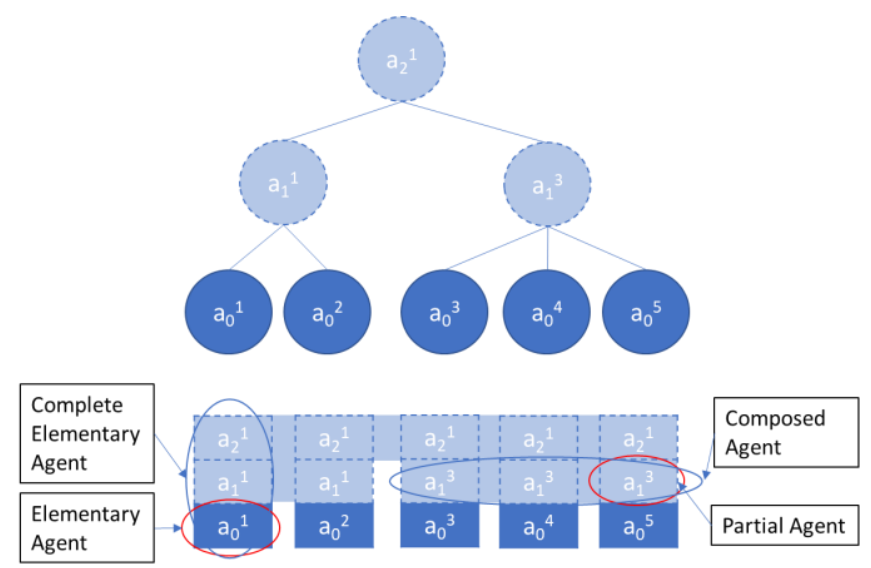

Fig. 2. Recursive agent compositions from (Hoang, 2012)

MAS is a framework to design overall control systems, but it is most of time employed to structure a little part of it (MAS for defining IP behavior for control in manufacturing or in logistics, MAS for controlling a specific feature of a WSN, ...). Currently, resource management services in existing WSN MAS solutions are tightly coupled with applications and generic resource management services still need to be developed. CM imply to control the devices network all along the lifecycle and to consider CM capacities to define new services. As demonstrated in section 2, many new services will depend on the product abstraction level. That is why a recursive and dynamic Holarchy (or agent-based model) to exploit CM capacities is needed. In this 
regard, the MAS-R model seems to be generic enough to be used as a basis to structure the Holonic Architecture needed for the Communicating Material.

\section{Structuration of a Holonic Architecture for the Communicating Material}

Section 2 states that a communicating material can be represented via a holarchy of product Holons but does not detail neither the structuration of the Holonic Architecture nor the rules necessary to undergo composition/decomposition operations. It is the objective of the current section.

A communicating material is structured as a holarchy composed of two types of Holons: the elementary material Holon and the composed material Holon. The elementary material Holon is the one connected with the real material. Indeed, we consider a communicating material as composed of elementary material elements not divisible, each equipped with one single electronic device that could have multiple functions (network communication, identification, sensing, ...). This electronic device represented the physical part of an elementary material Holon, called the Real Node afterwards (in reference with the wireless node used in some of our communicating material prototypes). This real node is in relation with a virtual node, called "Node Agent", representing the informational part of the elementary material Holon (fig. 3).

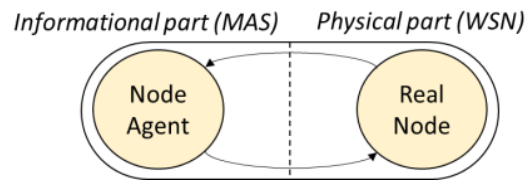

Fig. 3. Structure of an elementary material Holon

An aggregation of material Holons is called a composed Material Holons. In this holarchy, each Holon that is not elementary is a composed one. A representation of the internal structure of a composed material Holon is depicted fig. 4. A composed material Holons can also be an aggregation of other composed material Holons (linked denoted by (1) in the figure). Each material Holon belongs to a certain level in the Holarchy. The higher the level of holarchy, the higher abstract the material Holon is. The informational part of the composed material Holon is a composed agent. It is linked either to other composed agents or to node agents of elementary material Holons. Every agent can discuss with agents of its own level of hierarchy by exchanging classical messages (denoted by (2) in the figure).

Links between levels of the hierarchy are abstraction links (denoted by (3) in the figure). These are not classical messages but transformation functions, taking information of the lower level and transforming it in higher level information. To illustrate this proposal, this recursive architecture has been applied to a specific context, i.e. the project McBIM. This application is described in the next section. 


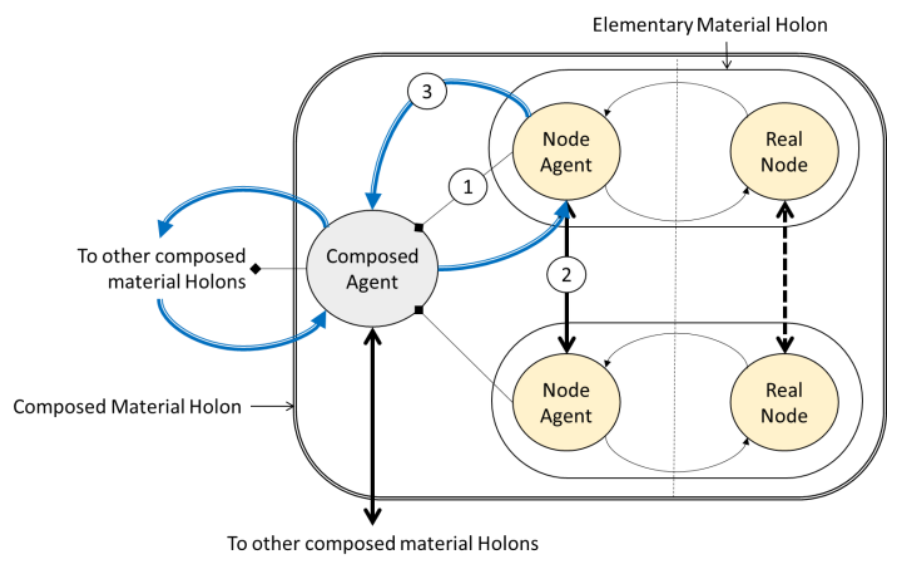

Fig. 4. Communicating Material recursive Holarchy

\section{$5 \quad$ Construction Industry Application}

\subsection{McBIM Project description}

The McBIM Project (Material communicating with the BIM - Building Information Modelling) (Derigent et al., 2019; McBIM Consortium, 2018) aims to design a "communicating concrete". This project is funded by the French National Research Agency and is coordinated by the CRAN with 2 other French laboratories and one company. CRAN works on the network and information management, LAAS designs the sensing and communicating nodes, LIB studies data interoperability and all these works are implemented by 360 SmartConnect/FINAO SAS.

The communicating concrete (see Figure 5) consists of a concrete structure where many sensing and communicating nodes are spread. The sensing nodes will periodically monitor the physical parameters (like temperature, humidity ...) of the concrete. Communicating nodes aggregate received data and transmit them to remote servers thanks to BIM standards. Besides, manufacturing data (like physical properties or information related to manufacturing actors) may also be considered. The communicating concretes behaviors may be different along its lifecycle. During the manufacturing phase, the WSN nodes are inserted and initialized. The communicating concretes periodically (by example every hour) monitor their physical status, store the physical propriety information and manufacturing actor information. During the construction phase, communicating concretes will be assembled. As communicating concretes arrive, auto-organization is then needed to dynamically define a 3D network to achieve energy savings. Concrete must frequently report its status to ensure the construction safety and updates the network organization. When the construction is completed, the 3D static WSN will regularly (every half-day) monitor structure health data (such as cracks, temperature, corrosion, etc.) to ensure the maintenance of the 
building. The communicating concrete element must last several decades from the manufacturing phase to the latest of the exploitation phase.

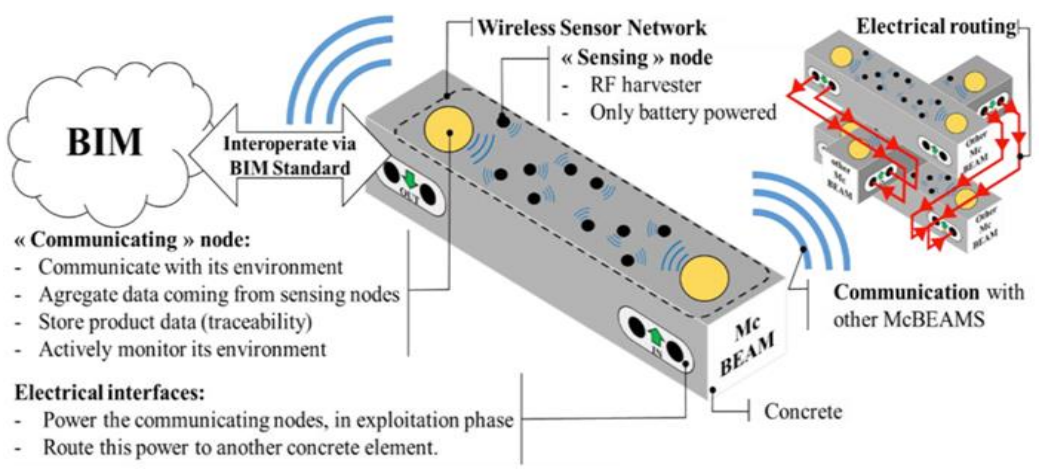

Fig. 5. Description of the ANR McBIM Project

\subsection{Communicating Concrete Control illustrations}

This part shows how we can use MAS proposal with the physical McBIM elements. As described in section 5.1, concrete pieces will pass through different phases (manufacturing, logistics, construction, exploitation), the frequencies which communicating concretes produce data must evolve over time. Because McBIM elements can interoperate each other, and in order to control the lifetime of the services provided by communicating concretes, the WSN have also to be reorganized. We focus on the energy control in the section.

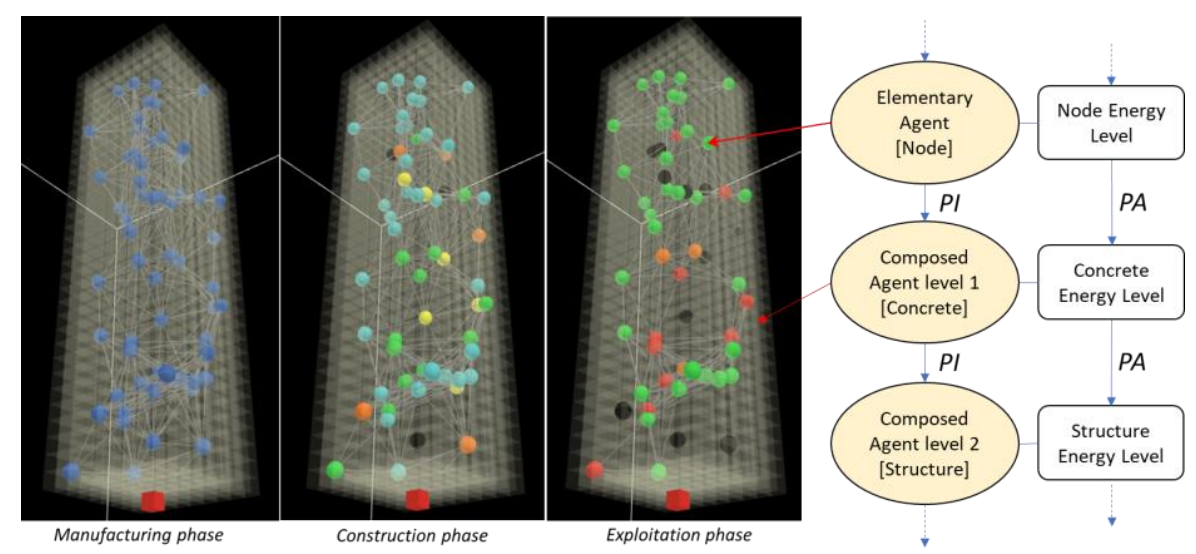

Fig. 6. Energy control and visualization of a concrete McBIM piece

We designed a 3D-energy estimation software on Netlogo platform. Netlogo is a multi-agent programmable modelling environment developed by Northwestern University (Wilensky, 1999). We developed this tool for integrating and storing sensor's values to be exploited by BIM applications. We model in real time energetic states of nodes 
on a mock-up representation (Digital Twin feature). Fig. 6 gives a view of the produced software for the different lifecycle phases of a McBIM concrete and the hierarchical and recursive corresponding MAS. In this application, elementary agents are composed of concrete bits in which nodes are inserted. There are called "nodes". Concrete parts like beams or walls are made of a composition of nodes and are called Concrete agents. Following our framework, these agents are composed agents of level 1. When concrete agents communicate, they can, if conditions are met, be grouped in higher level agents called Structure Agents.

In order to anticipate energy problems and to avoid stopping the services of a concrete McBIM element, we model at different abstraction levels (Node and Concrete for this example) the consumed energy. Blue color on a Node represent an energy level between 90 and 100\% (all nodes are blue during initialization and manufacturing phase). In construction or in exploitation phases, we can follow remaining energy of nodes represented by a color gradient. If too many nodes die (black color) or if BIM applications need to change the measurement frequency, the Structure agent can define another configuration for the WSN of the monitored McBIM element.



Fig. 7. Illustration of concrete pieces aggregation

The agent architecture has been modeled thanks to the framework describing the communicating material. Fig. 7 illustrates the aggregation ability bringing by our rescursive MAS. In a first step, concretes pieces $\mathrm{C}$ (green and red) each one composed by Elementary agents (E) have their own life. When Elementary Agent meet, they are automatically gathered in a Concrete Agent thanks to the PA operator. From that point, data generated by sensors are sent to the Concrete Agent thanks to the $P I$ operator. Indeed, temperatures monitored by each sensor are aggregated into a average temperature at the Concrete Agent level. In a second step, green and red sensor nodes (S) detect another concrete. In the MAS, this meeting leads to interactions between Elementary Agent (E) associated with different "Composed 
Agent level 1" (C1 and C2). Aggregation rules are shared by Aggregation model every agent $(\mathrm{E}, \mathrm{C}, \mathrm{MC}, \ldots)$ whatever its abstraction level and define if the green and red pieces have to be engaged in a relationship (case 2 and 3) or not (case 1: elementary agents interaction). If they are related, (C) Agents exchange to decide about the kind of the relationship. Green and red communicating concretes can be associated (case 2: only composed agents association). This case can represent temporary relationship (by example during storage or transport phase). In that case, this relation between agents of lower levels is then transformed into a higher relation between concrete agents thanks to $P O$ operator. In a more intensive relationship, the two McBIM pieces can aggregate their network, their models and theirs properties (case 3). This last case creates the new "Composed Agent level 2" (MC) thanks to the $P O$ operator followed by the $P A$ operator.

\section{Conclusion and perspectives}

This work formalizes the "Communicating Material" paradigm as an upgrade of "Intelligent Product" concept. A framework to design the product lifecycle monitoring of this new kind of object is studied. Inspired by holonic and Multi-Agent Systems approaches, a recursive MAS able to structure Communicating Materials is proposed. Aggregation and dynamic abilities are illustrated by application on McBIM project, whose main objective is the design of communicating concretes interacting during the overall lifecycle with BIM applications. The work of (Hoang, 2012) define 4 types of transformations functions, which could be considered as requirements when designing any type of recursive and dynamic Holarchies (not only Communicating Materials). To complete this work, transformation function and negotiation protocols still have to be clearly defined and instantiated on the McBIM Project.

\section{ACKNOWLEDGMENTS}

Authors thank financial support from the French National Research Agency (ANR) under the McBIM project, grant number ANR-17-CE10-0014.

\section{REFERENCES}

Botti, V., \& Giret, A. (2008). ANEMONA: A Multi-agent Methodology for Holonic Manufacturing Systems. Journal of Chemical Information and Modeling (Vol. 53). https://doi.org/10.1017/CBO9781107415324.004

Delgado, C. (2014). Organisation-based co-ordination of wireless sensor networks. University of Barcelone.

Demazeau, Y. (1995). From interactions to collective behaviour in agent-based systems. In In: Proceedings of the 1st. European Conference on Cognitive Science. Saint-Malo.

Derigent, W., David, M., Wan, H., Dragomirescu, D., Takacs, A., Loubet, G., ... Montegut, L. (2019). Materials communicating with the BIM: Aims and first results of the MCBIM project. In Structural Health Monitoring 2019: Enabling Intelligent Life-Cycle Health Management for Industry Internet of Things (IIOT) - Proceedings of the 12th 
International Workshop on Structural Health Monitoring. https://doi.org/10.12783/shm2019/32226

Hoang, T. (2012). Un modèle multi-agent récursif générique pour simplifier la supervision de systèmes complexes artificiels décentralisés. Université de Grenoble, Grenoble, France.

Koestler, A. (1967). The ghost in the machine. Hutchinson.

Koestler, Arthur. (1979). Janus: A summing up. Bulletin of the Atomic Scientists, 35(3), 4.

Kubler, S., Derigent, W., Thomas, A., \& Rondeau, E. (2014). Embedding data on «communicating materials » from context-sensitive information analysis. Journal of Intelligent Manufacturing, 25(5). https://doi.org/10.1007/s10845-013-0745-y

Kubler, Sylvain, Derigent, W., Thomas, A., \& Rondeau, É. (2010). Problem definition methodology for the «\{C\}ommunicating $\{\mathrm{M}\}$ aterial $»$ paradigm. In 10th IFAC Workshop on Intelligent Manufacturing Systems, Lisbon (Vol. 10, p. 198-203).

McBIM Consortium. (2018). WebPage of the McBIM Project. Consulté à l'adresse http://mcbim.cran.univ-lorraine.fr

McFarlane, D., Giannikas, V., Wong, A. C. Y., \& Harrison, M. (2013). Product intelligence in industrial control: Theory and practice. Annual Reviews in Control, 37(1), 69-88. https://doi.org/10.1016/j.arcontrol.2013.03.003

Mekki, K., Derigent, W., Zouinkhi, A., Rondeau, E., Thomas, A., \& Abdelkrim, M. N. (2016) Non-localized and localized data storage in large-scale communicating materials: Probabilistic and hop-counter approaches. Computer Standards and Interfaces, 44. https://doi.org/10.1016/j.csi.2015.08.010

Sallez, Y., Montreuil, B., \& Ballot, E. (2016). On the activeness of physical internet containers. Computers in Industry, 81, 96-104. https://doi.org/10.1007/978-3-319-15159-5_24

Ventä, O. (2007). Intelligent products and systems. Technology Theme-Final Report. VTT, Espoo: VTT Publications, 304.

Wilensky, U. (1999). NetLogo. http://ccl.northwestern.edu/netlogo/. Center for Connected Learning and ComputerBased Modeling Northwestern University Evanston IL.

Wong, C. Y., Mcfarlane, D., Zaharudin, A. A., \& Agarwal, V. (2002). The Intelligent Product Driven Supply Chain. In Proc. IEEE Int. Conf. on Systems, Man and Cybernetics (p. 4-6). 